\title{
Características de fluxo e projeto de tremonhas cônicas em silos verticais
}

\author{
José P. Lopes Neto $^{1}$ \& José W. B. do Nascimento ${ }^{1}$
}

\begin{abstract}
RESUMO
Para que projetos de silos verticais que envolvam armazenagem de produtos sólidos sejam seguros e confiáveis, é importante que se conheça o maior número de variáveis possíveis, tais como: propriedades de fluxo, geometria do silo e tipo de fluxo desejado. No intuito de validar as teorias de predição de fluxo e o dimensionamento de silos, foram determinadas as propriedades de fluxo de três produtos sólidos, analisadas e testadas as teorias de tipo de fluxo e de dimensionamento de tremonhas de Jenike e Enstad, respectivamente, além de comparados os resultados com os obtidos experimentalmente em modelo reduzido de silo de seção semicircular. Os resultados experimentais corroboram com a metodologia de tipo de fluxo de Jenike e de tremonhas cônicas de Enstad. Os valores de orifício de descarga calculados pelo método de Jenike se demonstraram superdimensionados, sendo $45 \%$ superior ao necessário para obtenção de fluxo.
\end{abstract}

Palavras-chave: teorias, dimensionamento, orifício, descarga

\section{Flow characteristics and design of conical hoppers in vertical silos}

\begin{abstract}
For design involving storage of bulk solids to be safe and reliable, it is important to know a large number of variables such as flow properties, geometry of silos and flow pattern. With the objective to validate prediction using flow theories and silo design procedures, the flow properties of three bulk solids were determined and Jenike's flow theory and Enstad's hopper design equations were applied and compared with those obtained experimentally in a reduced model of silo of semicircular section. The experimental results confirm the method of Jenike and Enstad for flow pattern and hopper design, respectively. The values of discharge orifice obtained by Jenike's equation are demonstrated to be oversized, being $45 \%$ higher than needed to provide flow.
\end{abstract}

Key words: theories, design, outlet, discharge 


\section{INTRODUÇÃO}

Pesquisas envolvendo o comportamento de produtos sólidos armazenáveis são, de certo modo, recentes, e carecem de maiores informações uma vez que novos procedimentos industriais são realizados em cada novo produto.

Segundo Lopes Neto et al. (2009a) dentre os principais obstáculos enfrentados pelas indústrias que manuseiam produtos granulares e pulverulentos se destacam a formação de arcos coesivos capazes de restringir, parcial ou completamente, a descarga cuja ocorrência está intrinsecamente relacionada às geometrias dos silos e das tremonhas, às propriedades físicas e de fluxo dos produtos sólidos e condições ambientais, tais como pressão, temperatura e umidade relativa.

Cannavacciuolo et al. (2009) afirmam que produtos pulverulentos e com alta coesão podem apresentar dificuldade de fluxo devido à influência das interações dinâmicas entre gás-sólido e sólido-sólido. Propõe-se, como forma de amenizar esta situação, a aeração do produto durante a descarga a fim de produzir gradientes de pressão perto do orifício de descarga.

Uma das grandes contribuições neste ramo de pesquisa foi dada por Jenike (1964) ao desenvolver o aparelho de cisalhamento direto por translação conhecido como Jenike ShearTester para a determinação do ângulo de atrito interno, ângulo de atrito com a parede, densidade consolidada, função fluxo e índice de fluxo, além de ter definido os padrões de fluxo em silos, de massa e funil, importantíssimo em projeto de silos verticais.

Schulze (2009) descreve que o Jenike ShearTester utiliza um conjunto de células para reproduzir o cisalhamento em amostras de produtos armazenáveis sendo possível, então, traçar círculos de tensão (círculos de Mohr) que representem graficamente o estado de tensão em uma porção do produto armazenado possibilitando a determinação das tensões de consolidação $\left(\sigma_{\mathrm{M}}\right)$, tensões não confinadas de deslizamento $\left(\mathrm{f}_{\mathrm{C}}\right)$ e do ângulo de atrito interno (Ø) de produtos armazenáveis (Lopes Neto et al., 2007).

Outra variável de grande relevância para a compreensão do comportamento de produtos em silos, é a chamada função fluxo (FF). Lopes Neto et al. (2009a) comentam que a função fluxo (FF) serve para estimar a capacidade dos sólidos em fluir por gravidade.

Juliano et al. (2006) afirmam que as propriedades de fluxo são responsáveis por determinar o tipo de fluxo em silos, tremonhas e em transportadores, sendo possível maximizar operações como descargas, transilagem, dosagem e empacotamento e ainda evitar inseguranças quanto ao tipo de fluxo e obstruções de descarga.

Ao investigar o fluxo de partículas de Politeleftalato de $3 \mathrm{~mm}$ de diâmetro em um silo com tremonha de $15^{\circ}$ com a vertical, Ostendorf \& Schwedes (2005) utilizaram a técnica de obtenção de imagens conhecida como Particle Image Velocimetry (PIV). Segundo os autores, foi possível definir o modelo de fluxo formado e os perfis de velocidade assumidos.

Ao aplicar a mesma técnica PIV, Slominski et al. (2007) puderam medir a deformação sofrida no produto, em função da rugosidade da parede do silo durante o descarregamento enquanto Sielamowicz et al. (2005) obtiveram uma sequência de 315 imagens do descarregamento de sementes de amaranto em que foi observada a evolução de um tipo intermediário de fluxo, o fluxo misto, durante a descarga por gravidade.

No desejo de compreender os critérios de fluxo, suas alterações e as variáveis interferentes, a presente pesquisa objetivou a determinação das propriedades de fluxo de três produtos armazenáveis (um granular e dois pulverulentos), a predição do tipo de fluxo e a validação das teorias, por meio de imagens digitais obtidas em modelo reduzido.

\section{Material e Métodos}

Como o escopo da pesquisa era estudar a armazenagem em silos, optou-se por utilizar três produtos empregados no meio agrícola, sendo escolhidos milho em grãos, milho triturado e farelo de soja.

A determinação da granulometria dos produtos analisados foi realizada pelo método clássico de peneiramento de amostra, utilizando $500 \mathrm{~g}$ cada um enquanto o teor de umidade foi encontrado pelo método Gravimétrico com secagem em estufa de $50 \mathrm{~g}$ de amostra de cada produto, a $105^{\circ} \mathrm{C}$ por $24 \mathrm{~h}$.

A determinação das propriedades de fluxo foi realizada utilizando-se o aparelho de cisalhamento direto translacional (Figura 1) e seguindo a metodologia descrita pela BMHB (1985). Foram utilizadas as séries $100 \mathrm{~N}(70,50$ e $35 \mathrm{~N}), 70$ $\mathrm{N}(50,35$ e $20 \mathrm{~N})$ e $50 \mathrm{~N}(35,20$ e $10 \mathrm{~N})$ como cargas de précisalhamento e de cisalhamento.

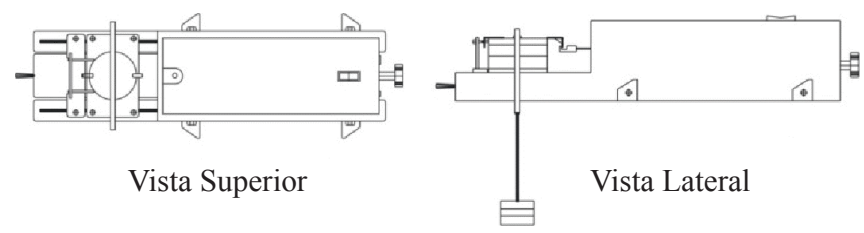

Figura 1. Aparelho de cisalhamento direto translacional

Com referência à determinação do ângulo de atrito com a parede $\left(\varnothing_{\mathrm{w}}\right)$ utilizou-se uma placa de aço rugoso, substituindo apenas o anel inferior pela amostra do material da parede enquanto os níveis de carga normais foram: 50, 40, 30, 20, 10 e 4,4 N. Todos os ensaios foram realizados apenas visando à obtenção das propriedades instantâneas de fluxo em temperatura ambiente $\left(24{ }^{\circ} \mathrm{C}\right)$ e umidade relativa do ar em torno de $67 \%$.

Jenike (1964) propôs o índice de fluabilidade (ff) como parâmetro de predição do comportamento do produto durante o descarregamento do silo obtido pela razão entre a tensão principal de consolidação, $\sigma_{\mathrm{M}}$, e a tensão não confinada de deslizamento, $\mathrm{f}_{\mathrm{C}}$, conforme Tabela 1.

Para determinação da função fluxo do produto $(\mathrm{FF})$ foram plotados os valores de $\sigma_{\mathrm{M}}$ versus $\mathrm{f}_{\mathrm{C}}$ obtidos através dos círculos de tensões de Mohr e, com a interceptação da curva de FF pela reta do fator fluxo da tremonha (ff), determinou-se a tensão

Tabela 1. Classificação do índice de fluabilidade

\begin{tabular}{cc}
\hline Comportamento do fluxo & Índice de fluabilidade $\left(\mathrm{ff}_{\mathrm{c}}\right)$ \\
Sem fluxo & $\mathrm{ff}_{\mathrm{c}}<1$ \\
Muito coesivo & $1<\mathrm{ff}_{\mathrm{c}}<2$ \\
Coesivo & $2<\mathrm{ff}_{\mathrm{c}}<4$ \\
Fácil & $4<\mathrm{ff}_{\mathrm{c}}<10$ \\
Livre & $\mathrm{ff}_{\mathrm{c}}>10$ \\
\hline
\end{tabular}


crítica não confinada $\left(\sigma_{\mathrm{CR}}\right)$ a fim de se evitar formação de arcos coesivos que provoquem obstruções durante a descarga. Tal metodologia é conhecida como critério fluxo - não fluxo de Jenike e define que, se a razão entre $\sigma_{M}$ e $f_{C}$ for maior que ff, haverá fluxo.

Quanto à determinação do fator fluxo da tremonha (ff) preferiu-se aplicar a Teoria Continuum Mechanics, proposta pelas Eqs. 1 a 5 de Enstad (1981):

$$
\begin{gathered}
\text { ff }=\frac{Y(1+\operatorname{sen} \delta)}{2(X-1) F(\theta) \cos \theta} \\
Y=\frac{(2+2 \cos \alpha)^{2}\left(\alpha^{1+\mathrm{m}}\right) \cos \theta+\operatorname{sen} \beta(\operatorname{sen} \alpha)^{1+\mathrm{m}}}{(1-\operatorname{sen} \delta)(\operatorname{sen} \alpha)^{2+\mathrm{m}}} \\
\mathrm{X}=\frac{2^{\mathrm{m}} \operatorname{sen} \delta}{1-\operatorname{sen} \delta}\left[\frac{\operatorname{sen}(\alpha+\beta)+\cos \theta}{\cos \theta}\right] \\
\mathrm{F}(\theta)=\left[\frac{65}{220-\theta}\right]^{\mathrm{m}}\left[\frac{200}{290-\theta}\right]^{1-\mathrm{m}}
\end{gathered}
$$

Pelas Eqs. 6 e 7 propostas por Enstad (1981) ainda foi possível determinar a inclinação mínima da tremonha com a horizontal $\left(\theta_{\mathrm{m}}\right)$ de forma a assegurar o fluxo.

$$
\begin{gathered}
\theta_{\mathrm{m}}=0,5 \cos ^{-1}\left(\frac{1-\operatorname{sen} \delta}{2 \operatorname{sen} \delta}\right)+\beta \\
\beta=\frac{\varnothing_{\mathrm{w}}+\operatorname{sen}^{-1}\left(\frac{\operatorname{sen} \varnothing_{\mathrm{w}}}{\operatorname{sen} \delta}\right)}{2}
\end{gathered}
$$

O orifício mínimo de descarga (D) foi calculado conforme proposto por Jenike (1964) através da Eq. 8.

$$
\mathrm{D}=\frac{\mathrm{H}(\theta) \sigma_{\mathrm{cr}}}{\gamma}
$$

em que:

D - abertura mínima do orifício, $m$

$\mathrm{H}(\theta)$ - constante igual a 2,3, adimensional

$\sigma_{\mathrm{CR}}$ - tensão crítica não confinada, $\mathrm{Pa}$

$\gamma$ - peso específico consolidado, $\mathrm{N} \mathrm{m}^{-3}$

Como uma das principais dificuldades no estudo do fluxo em silos é a impossibilidade de verificação in loco, devido à opacidade de suas paredes, optou-se por construir um silo metálico em modelo reduzido de secção transversal semicircular com a face frontal fechada por uma parede de vidro translúcido com $8 \mathrm{~mm}$ de espessura.

O modelo reduzido foi construído com paredes, fundo plano e tremonha em chapa metálica rugosa de espessura nominal de 1,2 $\mathrm{mm}$. Na primeira fase do experimento utilizou-se o fundo plano com orifício concêntrico de $60 \mathrm{~mm}$ de abertura (Figura 2A); em seguida, utilizou-se tremonha concêntrica e de inclinação com a horizontal de $70^{\circ}$ (Figura 2B) com orifício de descarga de mesmo diâmetro.
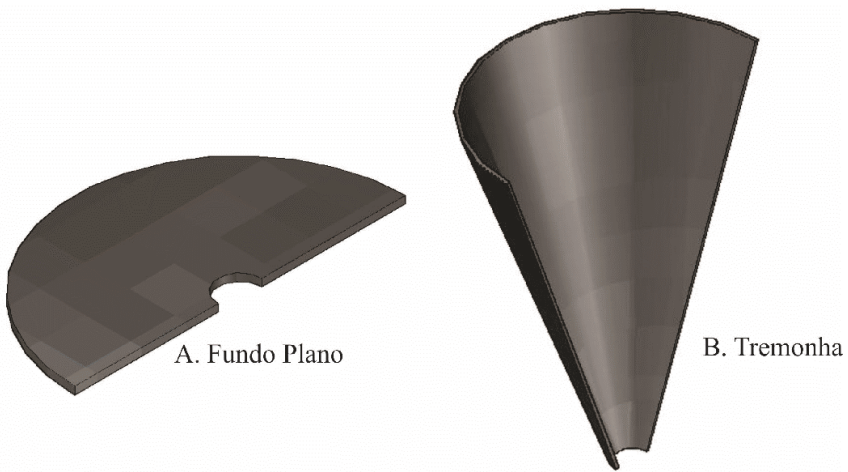

Figura 2. Fundo plano (A) e tremonha cônica (B) utilizados

O corpo do silo possuía as dimensões de 1,1 x 0,45 m (altura $\mathrm{x}$ diâmetro) representando um silo com relação $\mathrm{H} / \mathrm{D}$ igual a 2,4. As Figuras 3A e B representam o silo em modelo reduzido com a utilização do fundo plano, tremonha e a face frontal em vidro translúcido.
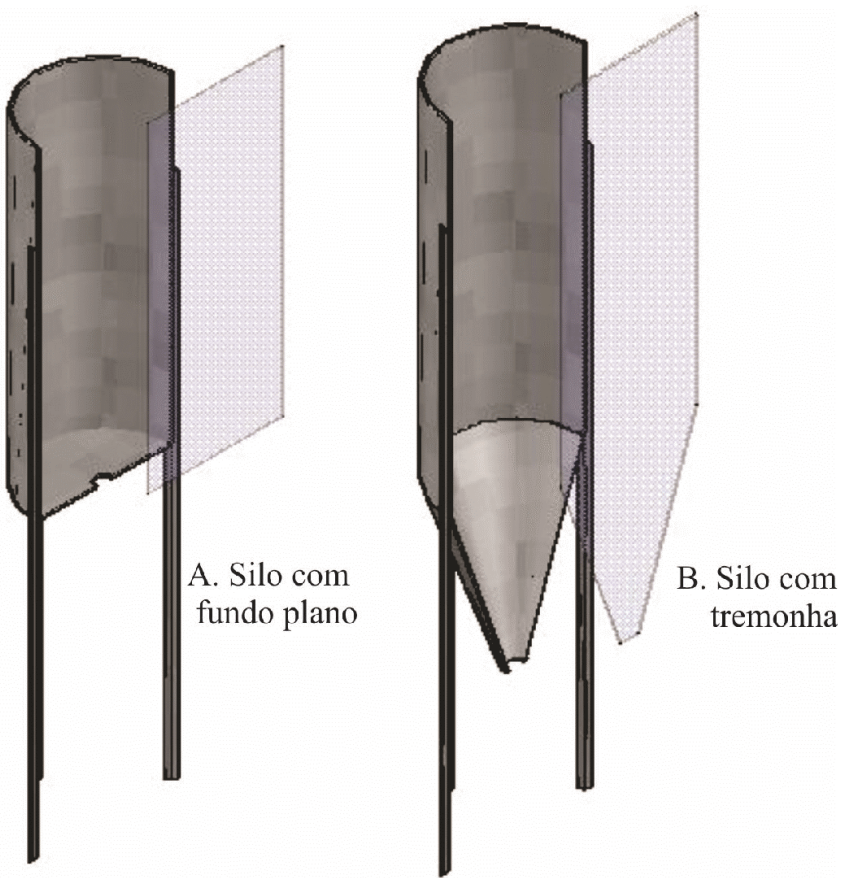

Figura 3. Silo com fundo plano (A) e tremonha (B)

Com vista à obtenção das imagens do perfil de fluxo, utilizou-se uma câmera digital de alta resolução posicionada a uma distância de 2,5 $\mathrm{m}$ do modelo reduzido. $\mathrm{O}$ procedimento experimental de fluxo consistiu em carregar o silo de forma manual, concêntrica e com velocidade aproximadamente constante, garantindo, assim, a mínima compactação possível devido ao impacto de queda do produto. Após um período de descanso de 30 min efetuouse o descarregamento do silo, por gravidade. Todos os ensaios foram realizados em quatro repetições a fim de se obter boa representatividade das informações coletadas. 


\section{Resultados E Discussão}

Na Figura 4 se encontram as curvas granulométricas dos três produtos analisados sendo possível perceber que o milho em grãos possui quase que a totalidade de suas partículas com dimensão imediatamente superior a 4,8 mm enquanto o milho triturado e o farelo de soja apresentam distribuição granulométrica mais uniforme nas demais peneiras.

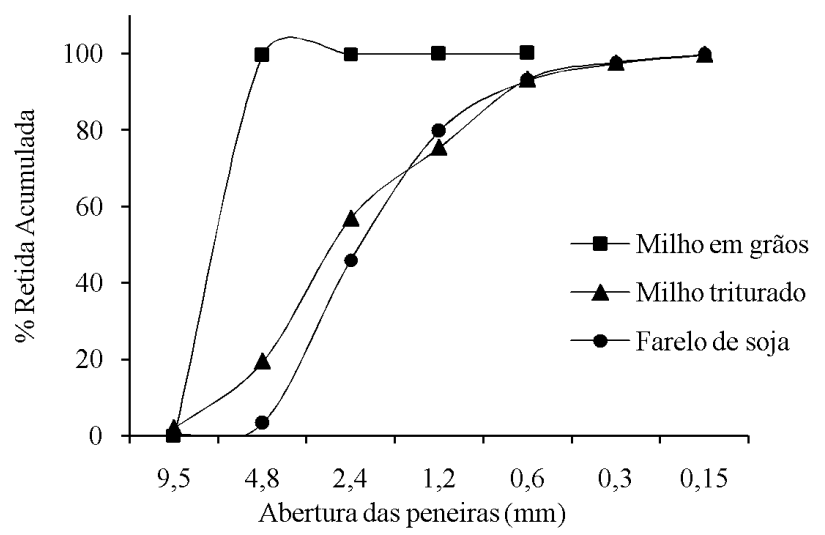

Figura 4. Curvas granulométricas dos produtos

Observa-se, ainda, que o milho triturado começa a acumular partículas a partir da peneira $2,4 \mathrm{~mm}$ e que, para o farelo de soja, se dá a partir da peneira 1,2 $\mathrm{mm}$ evidenciando uma ligeira diferença granulométrica entre os dois últimos produtos, nas peneiras de maior dimensão. Durante os ensaios foram registrados teores de umidade de 8,5, 6,6 e 6,5\% para milho em grãos, milho triturado e farelo de soja, respectivamente.

As propriedades de fluxo dos produtos se encontram na Tabela 2, na qual é importante destacar uma variação maior entre os limites inferior e superior, obtida para o ângulo de atrito interno $(\varnothing)$ do milho triturado, em torno de $31 \%$ enquanto que para os dois outros produtos esta variação não ultrapassou os $8 \%$.

Analisando apenas os valores absolutos desta propriedade, ressalta-se que o farelo de soja apresenta um plano de deslizamento mais inclinado (maiores limites para Ø) significando maior facilidade de cisalhamento e, por conseguinte, fluxo.

Outra variável significativa é a coesão de partículas (C) em que novamente se obteve o maior resultado para o milho triturado indicando tratar-se de um produto passivo de desenvolver alta coesão sob condição de armazenagem e, quando de seu descarregamento, é provável que problemas de obstrução sejam observados.

É interessante destacar, para os demais produtos, que o farelo de soja ainda possui coesão menor que para milho em grãos, fato este corroborado pelos maiores valores obtidos para o índice de fluxo e ângulos de atrito interno da soja triturada; entretanto, tal diferença na coesão pouco afeta a condição de fluxo para ambos os produtos sendo previsto um fluxo com característica livre, sem dificuldade de esvaziamento do silo.

Conforme Mohammed et al. (2011) a medição pela célula de Jenike da resistência à coesão de determinado produto pode ser feita apenas de forma indireta, através da extrapolação dos dados de tensão e, segundo Geldart et al. (2009) elevados valores de coesão podem refletir em baixa fluabilidade, o que confirma a relação de Coesão e ff $_{c}$ dos três produtos.

De acordo com a Tabela 2, a condição de fluabilidade mais crítica é referente ao milho triturado com índice $\mathrm{ff}_{c}$ igual a 3,8 representando, conforme Tabela 1, um produto com possibilidade de fluxo do tipo coesivo. O mesmo valor para o Índice de fluxo ( $\mathrm{ff}_{\mathrm{c}}$ ) foi encontrado por Lopes Neto et al. (2009b) ao analisarem amostras de ração avícola com teor de gordura de $8,3 \%$; portanto, para o dimensionamento do orifício de descarga deve-se considerar este valor a fim de se evitar a formação de arcos coesivos.

Ao aplicar a metodologia do Índice de fluabilidade ( $\mathrm{ff}_{\mathrm{c}}$ ) em produtos pulverulentos alimentícios sob armazenagem por 48 h, Lopes Neto et al. (2009a) encontraram valores 2 vezes superiores ao obtido para o milho triturado na condição instantânea, o que reforça a ideia de este ser um produto altamente susceptível à compactação no interior de silos.

As Figuras 5A, B e C representam as tensões de consolidação $\left(\sigma_{\mathrm{M}}\right)$ e tensões não confinadas de deslizamento $\left(\mathrm{f}_{\mathrm{C}}\right)$ para as séries de cargas de 100, 70 e $50 \mathrm{~N}$ para milho em grãos, milho triturado e farelo de soja, respectivamente. Observa-se que os maiores valores de $\sigma_{\mathrm{M}}$ foram obtidos para o farelo de soja (Figura $5 \mathrm{C}$ ) entre 23168,3 e 13307,2 $\mathrm{Pa}$, demonstrando que este produto desenvolve maior estado de tensão ativa; em contrapartida, para o mesmo produto foram observados os menores valores de $\mathrm{f}_{\mathrm{C}} \mathrm{o}$ que indica a necessidade de se atingir baixos valores de tensão para ocorrer o cisalhamento da massa.

Ao construir os círculos de Mohr para o milho triturado, Figura 5B, foram encontradas as menores diferenças entre tensão de consolidação $\left(\sigma_{\mathrm{M}}\right)$ e tensão não confinada de deslizamento $\left(\mathrm{f}_{\mathrm{C}}\right)$ o que indica, sobremaneira, que a mudança de estado ativo para o passivo se dá em menor intensidade dificultando a ocorrência de cisalhamento da massa e, portanto, desfavorecendo o fluxo. A Figura 5A demonstra uma situação intermediária já evidenciada pelo índice $\mathrm{ff}_{\mathrm{c}}$ proposto por Jenike (1964).

A relação entre tensão de consolidação $\left(\sigma_{\mathrm{M}}\right)$ e tensão não confinada de deslizamento $\left(\mathrm{f}_{\mathrm{C}}\right)$, chamada yieldlocus, significa a potencialidade do produto em fluir (Opalinski et al., 2012) e representa a máxima tensão de cisalhamento capaz de ser suportada pelo produto sob dada tensão normal (Ripp \& Ripperger, 2010) podendo, ainda, ser afetada por diversos fatores, como umidade, dimensão de partículas, temperatura e tempo de armazenagem.

Tabela 2. Propriedades de fluxo dos produtos

\begin{tabular}{|c|c|c|c|c|c|c|c|c|c|c|}
\hline \multirow{2}{*}{ Produto } & \multicolumn{2}{|c|}{$\gamma$} & \multicolumn{2}{|c|}{$\emptyset$} & \multicolumn{2}{|c|}{$\delta$} & \multicolumn{2}{|c|}{$\emptyset_{w}$} & \multirow{2}{*}{ C } & \multirow{2}{*}{$\mathrm{ff}_{\mathrm{c}}$} \\
\hline & Inf & Sup & Inf & Sup & Inf & Sup & Inf & Sup & & \\
\hline Milho em grãos & 7395,1 & 7405,5 & 21,1 & 22,9 & 23,9 & 25,0 & 13,2 & 15,8 & 400,6 & 13,6 \\
\hline Milho triturado & 7441,4 & 7638,1 & 25,3 & 33,3 & 34,3 & 38,8 & 23,0 & 23,9 & 935,3 & 3,8 \\
\hline Farelo de soja & 6564,2 & 6640,1 & 32,8 & 35,1 & 34,1 & 35,5 & 23,4 & 25,0 & 128,0 & 54,3 \\
\hline
\end{tabular}

$\gamma$ - peso específico consolidado $\left(\mathrm{N} \mathrm{m}^{-3}\right) ; \varnothing$ - ângulo de atrito interno $\left({ }^{\circ}\right) ; \delta$ - efetivo ângulo de atrito interno $\left({ }^{\circ}\right) ; \varnothing_{w}$ - ângulo de atrito interno com parede de aço rugoso $\left({ }^{\circ}\right) ; C$ - média da coesão de partículas $(\mathrm{Pa})$; ff - média do Índice de fluxo de Jenike; Inf, Sup - limites inferior e superior, respectivamente 
A.

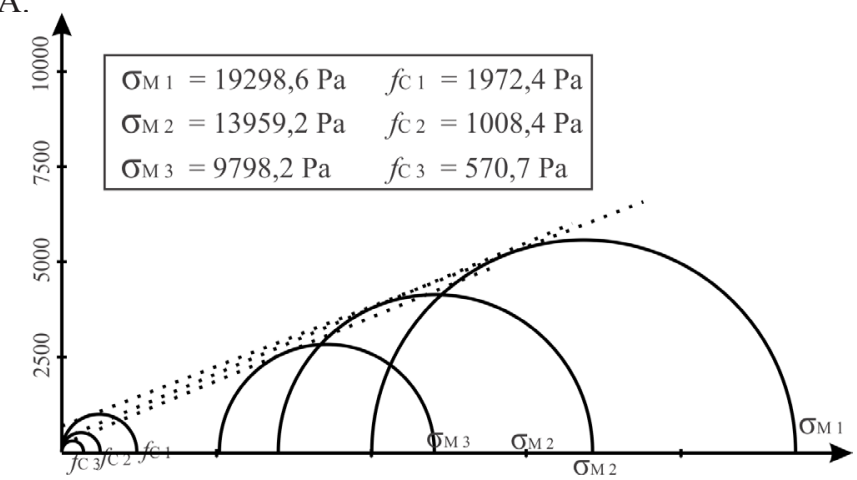

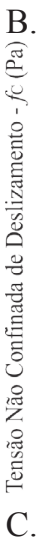
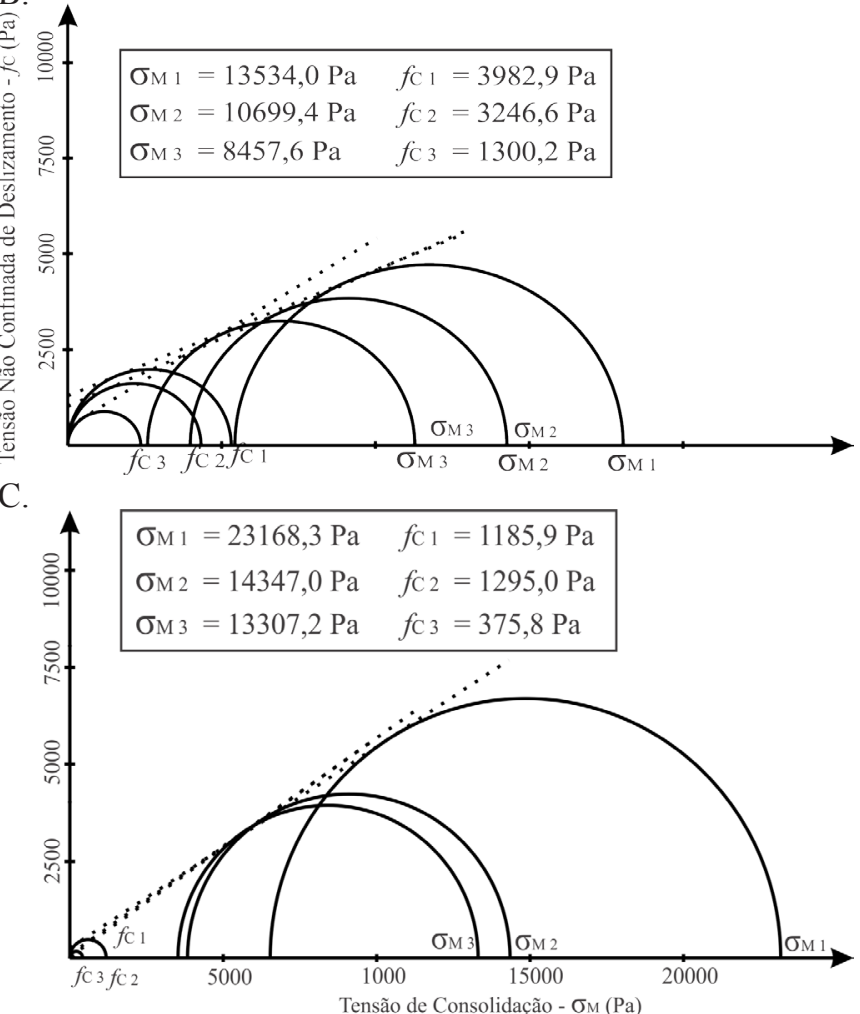

Figura 5. Círculos de tensões de Mohr. (A) Milho em grãos, (B) Milho triturado (C) Farelo de soja

As curvas representativas da função fluxo (FF) são encontradas na Figura 6 com a maior inclinação sendo representada para o milho triturado correspondendo ao seu baixo índice de fluabilidade e difícil descarregamento. Ganesan et al. (2008) comentam que o comportamento da função fluxo (FF) é resultado de uma combinação entre as propriedades

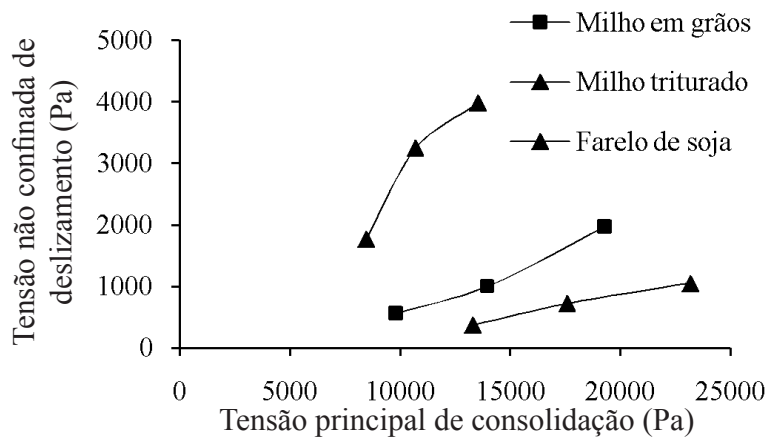

Figura 6. Função fluxo dos produtos físicas e de fluxo, de condições ambientais e dos equipamentos utilizados para manusear, armazenar e processar esses produtos.

Sabe-se que a curva mais próxima ao eixo horizontal representa um produto de fluxo fácil e, seguindo uma direção anti-horária, tal produto tende a apresentar maior resistência ao escoamento (Lopes Neto et al., 2007) o que evidencia a condição de fluxo livre para o milho em grãos e farelo de soja.

$\mathrm{Na}$ Tabela 3 se encontram as dimensões geométricas da tremonha cônica, podendo-se ressaltar uma inclinação máxima com a horizontal de $70,2^{\circ}$ para o farelo de soja seguido do milho triturado com $68,4^{\circ}$. O maior valor de ff foi obtido para o milho em grãos $(2,73)$ sinalizando menor eficiência da tremonha em promover o fluxo, juntamente com a maior abertura de descarga encontrada $(0,16 \mathrm{~m})$. Para o milho triturado não foi possível determinar uma abertura mínima de descarga pela metodologia aplicada nos ensaios.

Tabela 3. Características geométricas da tremonha

\begin{tabular}{ccccc}
\hline Produto & ff & $\begin{array}{c}\text { Tensão } \\
\text { crítica (Pa) }\end{array}$ & $\mathbf{D}(\mathbf{m})$ & $\boldsymbol{\theta}_{\mathbf{m}}\left({ }^{\circ}\right)$ \\
Milho em grão & 2,73 & 503,8 & 0,16 & 51,3 \\
Milho triturado & 1,48 & -- & -- & 68,4 \\
Farelo de soja & 1,59 & 316,2 & 0,11 & 70,2 \\
\hline
\end{tabular}

Matchett et al. (2009) analisaram a formulação de Jenike (1964) e encontraram valores de orifício de descarga superestimados em até $100 \%$ quando comparados aos dos experimentos reais e concluíram que o valor de $\mathrm{f}_{\mathrm{C}}$ baseado no modelo de Berry-Jenike produz dimensões de orifício de descarga mais perto da realidade.

São mostradas, a seguir, as Figuras 7A, B e C, referentes aos ensaios de fluxo em silo com fundo plano para milho em grãos, milho triturado e farelo de soja, respectivamente. Na Figura 7A é interessante destacar que, inicialmente, ocorreu a formação do fluxo de massa com todo produto em movimento descendente, até atingir uma relação altura/diâmetro (H/D) aproximada de 1,3 em que, a partir de então, predominou o fluxo de funil sendo caracterizado por uma porção parada do produto enquanto outra se encontrava em movimento.

Esta condição é conhecida como fluxo misto e, segundo Sadowski \& Rotter (2011), é caracterizada quando os limites do canal de fluxo chegam a interceptar as paredes do silo ainda durante o processo de descarregamento e são característicos de silos classificados esbeltos, cuja relação H/D é maior ou igual a 2 .

Ressalta-se também que a mudança do tipo de fluxo pode ser observada pela variação da forma da superfície do produto no interior do silo tendo a forma convexa no início do descarregamento passando a se nivelar no momento em que ocorre mudança de tipo de fluxo finalizando com a forma de um cone invertido com angulação aproximada de $45^{\circ}$.

$\mathrm{Na}$ Figura $7 \mathrm{~B}$ se encontram as imagens do milho triturado. Foi conveniente uma interferência manual com objetivo de romper o arco coesivo, visto que impossibilitava o produto de fluir. É importante notar que a forma de obstrução ocorrida não seguiu estritamente o predito pelas teorias de arcos coesivos assumindo, neste caso, uma forma mais cilíndrica. 
A.

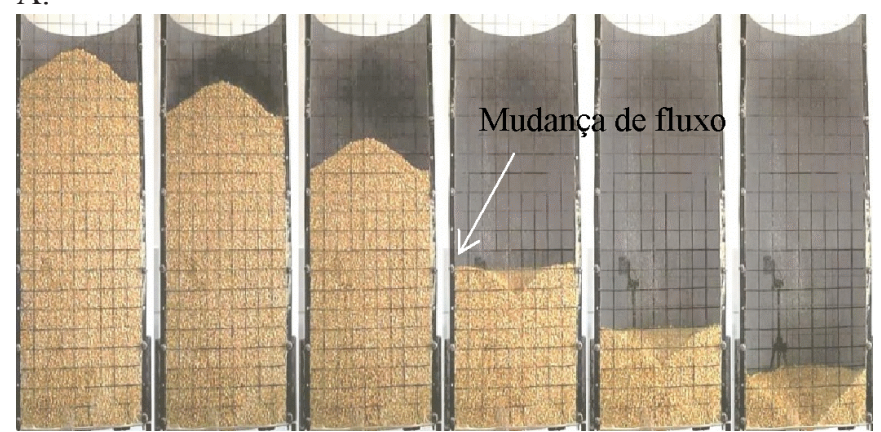

B.

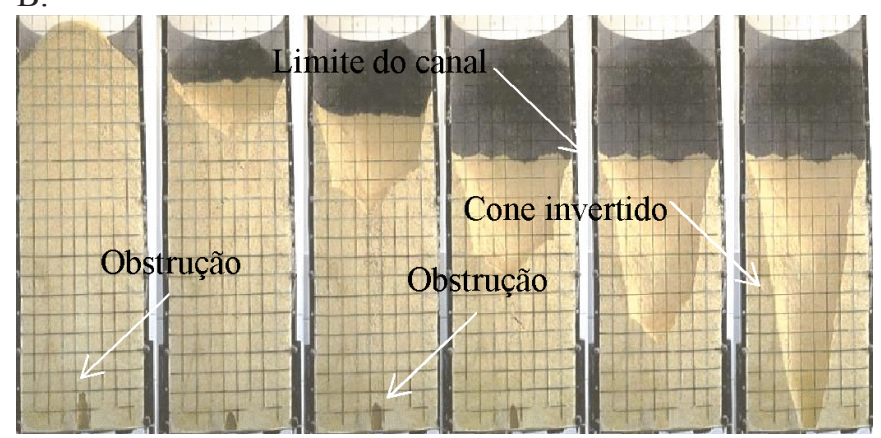

C.

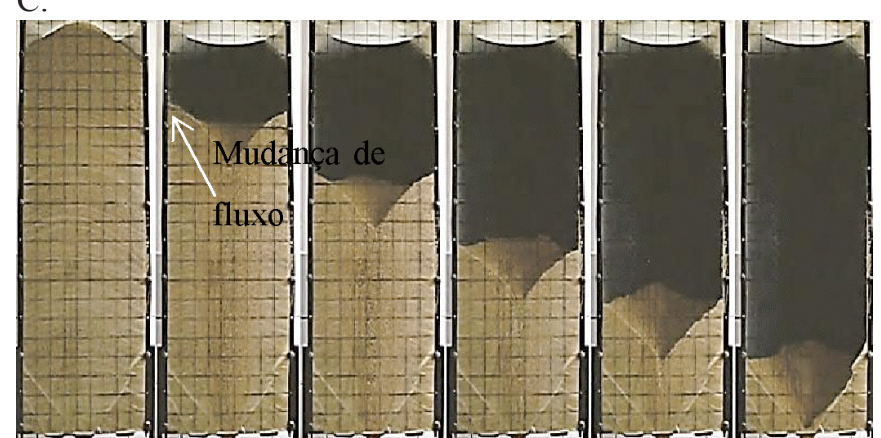

Figura 7. Descarregamento com fundo plano. (A) Milho em grão, (B) Milho triturado e (C) Farelo de soja

Matchett (2007) concluiu que, apesar de diversas teorias de formação de arcos coesivos, como as de Jenike (1964), Enstad (1981) e Walker (1966) previrem a obstrução na forma de arcos circulares e, ainda, terem boa aproximação quando comparadas a situações reais, isto não significa que a hipótese de arco circular é válida para qualquer situação de descarga, pelo fato de não haver, ainda, informações experimentais das tensões no produto localizado próximo ao orifício de descarga.

Rompida a obstrução, o descarregamento ocorreu por um canal de fluxo na forma de cone invertido no momento em que seus limites atingiram a relação $\mathrm{H} / \mathrm{D}=1,5$ e todo o processo se deu por meio de sucessivas interrupções com a formação de arcos que interrompiam a descarga.

A Figura 7C demonstra a mudança de fluxo nos momentos iniciais do descarregamento, relação $\mathrm{H} / \mathrm{D}=2$, com permanência do fluxo de funil deste ponto até o final do processo de descarga finalizando na formação do talude com ângulo aproximado de $54^{\circ}$ com a horizontal.

Nas Figuras 8A, B e C se acham as imagens sequenciais de descarregamento de milho em grãos, milho triturado e farelo de soja, respectivamente, em silo com tremonha. A Figura 8A demonstra que o fluxo desenvolvido no descarregamento de
B.

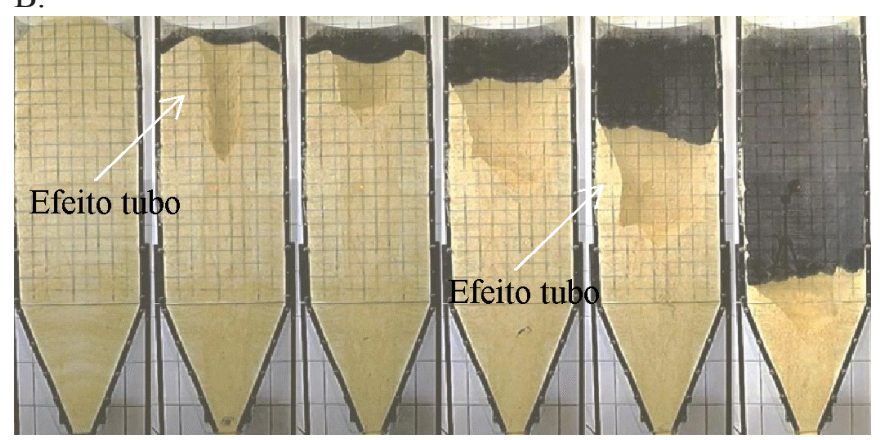

C.

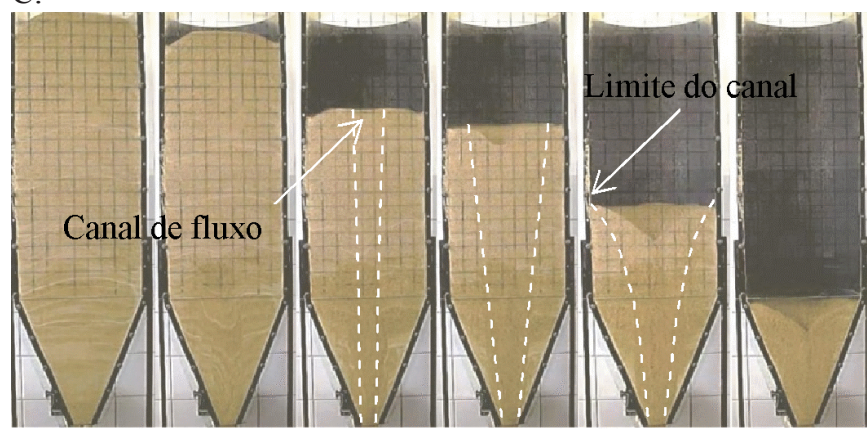

Figura 8. Descarregamento com tremonha. (A) Milho em grão, (B) Milho triturado e (C) Farelo de soja

milho em grãos foi o fluxo de massa clássico com a permanência da forma convexa da superfície superior do produto, até atingir a zona de transição corpo do silo-tremonha confirmando a predição quanto ao tipo de fluxo e eficiência da tremonha cônica.

O fluxo de massa apresenta algumas vantagens em relação ao fluxo de funil, principalmente quando se deseja evitar flutuações na vazão de descarga e reduzir os efeitos de segregação, melhorando a homogeneidade; em contrapartida, apresentam as desvantagens de ocorrer elevadas tensões na zona de transição e de maior desgaste superficial das paredes, devido ao atrito constante (Calil Júnior \& Cheung, 2007).

Percebe-se, na Figura 8B, que houve descarregamento do milho triturado, mesmo sendo de forma desordenada, mal estruturada e de difícil definição confirmando a previsão de um fluxo coesivo pelo índice de fluxo (ff) de Jenike (1964). Foi possível constatar visualmente que o fluxo se iniciou com a formação de um canal de fluxo vertical e central propagandose no sentido ascendente, até atingir a superfície superior da massa armazenada. Notou-se, também, que o descarregamento se deu através de sucessivas tentativas de formação do efeito tubo e frequentes desmoronamentos das paredes laterais, sempre quando atingiam uma altura de formação média de $45 \mathrm{~cm}$. 
Para a Figura 8C observou-se o fluxo do tipo massa durante todas as repetições mas com pequena variação quando comparado ao tipo clássico da Figura 8A. No descarregamento de farelo de soja (Figura 8C) o fluxo de massa clássico ocorreu até uma relação $\mathrm{H} / \mathrm{D}=1,5$ quando houve a distinção de um canal de fluxo mais veloz com limites periféricos atingindo as paredes verticais a uma relação $\mathrm{H} / \mathrm{D}=0,9$.

Um ponto importante a ser levantado é a possibilidade da existência da parede frontal de vidro ter afetado o produto durante seu descarregamento em razão da sua superfície mais lisa; acredita-se, contudo, que esta interferência tenha ocorrido apenas nos vetores de velocidade do fluxo e não no perfil de fluxo e suas particularidades.

Apesar das variações do fluxo de massa demonstradas nas Figuras 8A, B e C, a inclinação da tremonha foi suficiente para promover o esvaziamento do silo por completo validando as equações propostas por Enstad (1981) para esta finalidade. Quanto ao orifício de descarga a metodologia aplicada apresentou-se conservadora ao recomendar orifícios com dimensões 1,8 a 2,6 vezes maiores que os utilizados neste estudo, para obtenção do fluxo.

\section{Conclusões}

1. O modelo de predição pelo Índice de fluxo (ff $)$ de Jenike (1964) previu com exatidão o modo como o fluxo dos três produtos se daria nas duas situações de descarregamento.

2. A teoria de dimensionamento da geometria da tremonha proposta por Enstad (1981) demonstrou-se adequada ao recomendar uma inclinação suficiente para assegurar a ocorrência de fluxo de massa e o descarregamento completo dos produtos analisados.

3. Os valores teóricos para o orifício de descarga apresentaram grande variação quando comparados ao orifício testado demonstrando a necessidade de uma adequação da equação proposta.

4. O modelo reduzido de forma semicircular demonstrou-se adequado para a visualização do perfil de fluxo e das variações ocorrentes durante o fluxo.

\section{Agradecimentos}

Os autores agradecem ao Grupo de Estudos em Silos (SILOS) e ao Grupo de Estudos em Construções Rurais e Ambiência (GCAMB) da Universidade Federal de Campina Grande, pelo apoio em prol da realização desta pesquisa.

\section{Literatura Citada}

BMHB - British Materials Handling Board. Draft code of practice for the design of silos, bins, bunkers and hoppers. Berkshire: BMHB, 1985. 143p.

Calil Júnior, C.; Cheung, A. B. Silos: Pressões, fluxo, recomendações para o projeto e exemplos de cálculo. São Carlos: EESC. 2007. 232p.

Cannavacciuolo, A.; Barlleta, D.; Donsì, G.; Ferrari, G.; Poletto, M. Arch-free flow in aerated silo discharge of cohesive powders. Powder Technology, v.191, p.272-279, 2009.
Enstad, G. G. A novel theory on the arching and doming in mass flow hoppers. Bergen: The Michelsen Institute, 1981. 172p.

Ganesan, V.; Rosentrater, K. A.; Muthukumarappana, K. Flowability and handling characteristics of bulk solids and powders - A review with implications for DDGS. Biosystems Engineering, v.101, p.425-435, 2008.

Geldart, D.; Abdullah, E. C.; Verlinden, A. Characterisation of dry powders. Powder Technology, v.190, p.70-74, 2009.

Jenike, A. W. Storage and flow of silos. Bulletin 123. Salt Lake City: University of Utah, 1964. 89p.

Juliano, P.; Muhunthan, B.; Cánovas, G. V. B. Flow and shear descriptors of preconsolidated food powders. Journal of Food Engineering, v.72, p.157-166, 2006.

Lopes Neto, J. P.; Silva, V. R. da; Nascimento, J. W. B. do. Propriedades de fluxo de produtos pulverulentos alimentícios. Revista Brasileira de Engenharia Agrícola e Ambiental, v.13, p.639-644, 2009a.

Lopes Neto, J. P.; Nascimento, J. W. B. do; Silva, V. R. da. Efeito do tempo de armazenagem de rações avícolas no dimensionamento de silos. Engenharia Agrícola, v.29, p.518-527, 2009b.

Lopes Neto, J. P.; Nascimento, J. W. B. do; Silva, V. R. da; Lopes, F. F. M. Propriedade de fluxo e característica de escoabilidade de rações avícolas para dimensionamento de silos. Revista Ciência e Agrotecnologia, v.31, p.851-859, 2007.

Matchett, A. J. The shape of the cohesive arch in hoppers and silos - Some theoretical considerations.Powder Technology, v.171, p.133-145, 2007.

Matchett, A. J.; O’Neil, J.; Shaw, A. P. Stresses in bulk solids in wedge hoppers: A flexible formulation of the co-ordinate specific, Lame-Maxwell equations for circular arc, principal stress systems. Powder Technology, v.194, p.166-180, 2009.

Mohammed, S. A.; Abdullah, E. C.; Geldart, D.; Raman, A. A. A. Measuring powder flowability with a modified Warren Spring cohesion tester. Particuology, v.9, p.148-154, 2011.

Opalinski, I; Chutkowski, M.; Stasiak, M. Characterizing moist food-powder flowability using a Jenike shear-tester. Journal of Food Engineering, v.108, p.51-58, 2012.

Ostendorf, M.; Schwedes, J. Application of particle image velocimetry for velocity measurements during silo discharge. Powder Technology, v.158, p.69-75, 2005.

Ripp, M.; Ripperger, S. Influence of temperature on the flow properties of bulk solids. Chemical Engineering Science, v.65, p.4007-4013, 2010.

Sadowski, A. J.; Rotter, J. M. Steel silos with different aspect ratios: II - behaviour under eccentric discharge. Journal of Constructional Steel Research, v.67, p.1545-1553, 2011.

Schulze, D. Powders and bulk solids. Behavior, characterization, storage and flow. 1.ed. Berlin: Springer, 2009. 517p.

Sielmamowicz, I.; Blonski, S.; Kowalewski, T. A. Optical technique DPIV in measurements of granular material flows, Part 1 of 3 - Plane hoppers. Chemical Engineering Science, v.60, p.589-598, 2005.

Slominski, C.; Niedostatkiewicz, M.; Tejchman, J. Application of particle image velocimetry (PIV) for deformation measurement during granular silo flow. Powder Technology, v.173, p.1-18, 2007.

Walker, D. M. An approximate theory for pressures and arching in hoppers. Chemical Engineering Science, v.21, p.975-997, 1966. 